Received: February 09, 2016 Accepted: February 20, 2017 Published: March 03, 2017

\section{Effectiveness and Safety of Direct Oral Anticoagulants Compared With Vitamin K Antagonists in Non-Valvular Atrial Fibrillation Patients Undergoing Electrical Car- dioversion}

\author{
Luna Carrillo-Alemán*, Elena Candela-Sánchez, Teresa Lozano, Juan Gabriel Martínez- \\ Martínez, Francisco Sogorb-Garri, Amaya García-Fernández
}

Department of Cardiology, General University Hospital of Alicante, Alicante Institute for Health and Biomedical Research (ISABIAL - FISABIO Foundation), Alicante, Spain

*Corresponding author: Luna Carrillo-Alemán , Hospital General Universitario de Alicante, C/ Pintor Baeza, sn. 5o planta, Cardiología, CP 03010, Alicante, Spain, Tel: 0034 620556167; E-mail: lunicarrillo@gmail.com

\section{Abstract}

Introduction: Direct Oral Anticoagulants (DOACs) have positioned themselves as an effective therapy in Non-Valvular Atrial Fibrillation (NVAF). Recently, they have been approved for use in Electrical Cardioversion (ECV). Our aim was to assess the effectiveness and safety of DOACs in this context, compared to Vitamin K Antagonists (VKAs).

Methods: Observational, retrospective study of consecutive patients with NVAF undergoing a scheduled ECV under oral anticoagulants (DOACs or VKAs). Thromboembolic events, bleeding and death were registered on the first month after ECV and at one-year follow-up.

Results: Between june 2012 and june 2014, 342 patients underwent ECV. In 138 patients (40.4\%) ECV was performed with DOACs, and in the remaining 204 (59.6\%), under VKAs. No thromboembolic events associated with ECV (at 30 days) were documented in none of the groups. At one year follow-up, no patient with DOACs suffered thromboembolic events, compared to 3 (1.3\%) with VKAs $(\mathrm{p}=0.828)$. Seven $(5.8 \%)$ patients with DOACs and $27(14.5 \%)$ with VKAs $(\mathrm{p}=0.017)$ presented bleeding events. None of the patients experienced intracranial bleeding. Two patients (1.4\%) under DOACs and 4 (2.0\%) under VKAs died during follow-up.
Conclusions: In real-world NVAF patients undergoing ECV, DOACs are effective and safe, both short and midterm after the procedure.

\section{Keywords:}

Atrial fibrillation; Cardioversion; Anticoagulants; Embolism; Hemorrhage;

\section{Abbreviations}

DOAC: Direct Oral Anticoagulants

VKA: Vitamin K Antagonists

ECV: Electrical Cardioversion

NVAF: Non-Valvular Atrial Fibrillation

\section{Introduction}

Direct oral anticoagulants (DOACs) have been shown to have at least the same efficacy and a better safety profile than vitamin $\mathrm{K}$ antagonists (VKAs) in patients with non-valvular atrial fibrillation (NVAF). The subanalysis of patients undergoing electrical cardioversion (ECV) in the large pivotal studies of DOACs in NVAF suggests that these drugs are also effective and safe in this field $[1,2]$. Recently, the X-VeRT study demonstrated an efficacy and safety of rivaroxaban similar to warfarin in individuals undergoing ECV [3]. In addition, these data are supported by some realworld observational studies [4].

The main objective of our study was to analyze the efficacy (incidence of thrombotic complications and mortality) and safety (hemorrhagic events) of DOACs compared to VKAs in a real-life population of NVAF patients undergoing ECV. 


\section{Patients and Methods}

We performed an observational, retrospective study on the evolution of a cohort of patients with NVAF undergoing ECV in a tertiary hospital in Spain. All patients received anticoagulant treatment before ECV for at least 3 weeks, as indicated by clinical practice guidelines [5]. The choice of the type of anticoagulant treatment was decided by the responsible physician before the patient was referred to the Arrhythmia Unit for ECV. In order to perform the ECV, in patients with VKAs, international normalized ratio (INR) values in therapeutic range (between 2 and 3 ) were required during the previous 3 weeks, and in those who received DOACs, therapeutic compliance was required (at least $80 \%$ of dose).

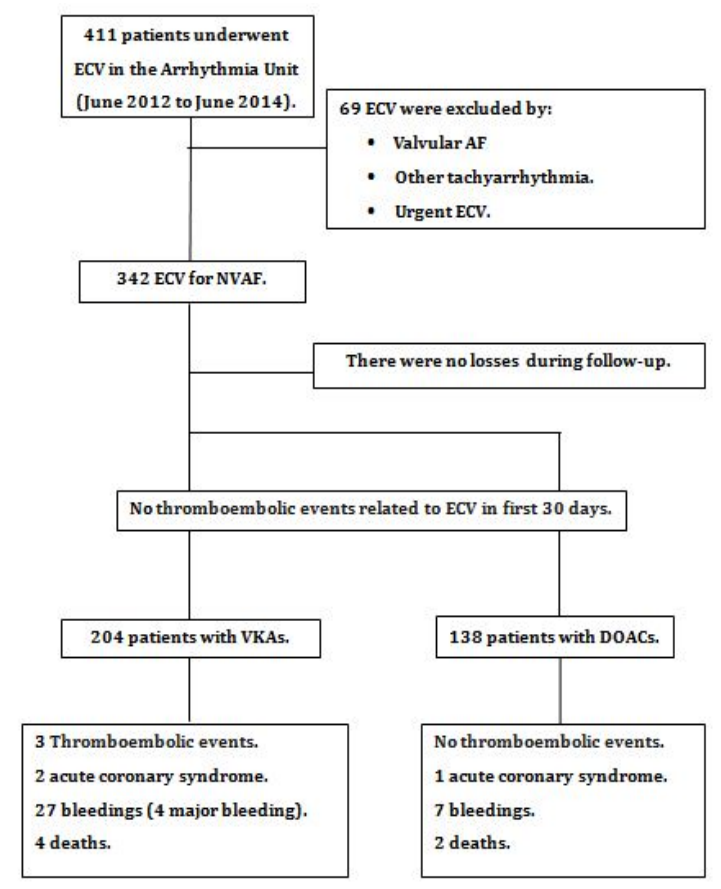

Figure 1: Flow chart of patients

After the ECV, the occurrence of thromboembolic events, acute coronary syndrome, mortality and hemorrhages, both on the first 30 days after the procedure and at one year follow-up was evaluated. This study was approved by the Ethical Committee of Alicante University Hospital and was performed in accordance with the ethical standards laid down in the 1964 Declaration of Helsinki and its later amendments. Patients gave their informed consent prior to their inclusion in the study.

Regarding statistical analysis, quantitative variables are expressed as mean \pm standard deviation or median (IQR: interquartile range). Qualitative variables, as absolute and relative frequencies. Continuous variables were analyzed to verify a normal distribution using the Kolmogorov-Smirnov test. Comparison between variables was performed using Pearson's Ji2 test, Student's $\mathrm{T}$ test and Log rank test. Hazard ratio (HR) and the $95 \%$ confidence intervals (CI) were calculated from the estimated parameters by the regression model. A value of $\mathrm{p}<0.05$ was considered statistically significant. Data were analyzed using the statistical
Table 1 Baseline Characteristics of Patients

\begin{tabular}{|c|c|c|c|}
\hline \multirow{2}{*}{$\begin{array}{c}\text { Baseline } \\
\text { characteristics }\end{array}$} & VKAs & DOACs & \multirow{2}{*}{$\mathrm{p}$} \\
\hline & $(n=204)$ & $(n=138)$ & \\
\hline Age, years & $66.8 \pm 11.1$ & $62.5 \pm 12.3$ & 0.001 \\
\hline Men & $143(70.1)$ & $105(76.1)$ & 0.13 \\
\hline CHA2DS2VASc & $3(1-4)$ & $2(1-3)$ & 0.001 \\
\hline HASBLED & $2(1-3)$ & $1(1-3)$ & 0.001 \\
\hline $\begin{array}{c}\text { Arterial } \\
\text { hypertension }\end{array}$ & $145(71.1)$ & $78(56.5)$ & 0.006 \\
\hline Diabetes Mellitus & $46(22.5)$ & $21(15.2)$ & 0.094 \\
\hline Heart failure & $69(33.8)$ & $23(16.7)$ & 0.001 \\
\hline $\begin{array}{c}\text { Concomitant heart } \\
\text { disease }\end{array}$ & $69(33.8)$ & $36(26.1)$ & 0.128 \\
\hline Active smoking & $37(20.6)$ & $22(17.6)$ & 0.52 \\
\hline Previous stroke & $26(12.7)$ & $9(6.5)$ & 0.062 \\
\hline Antiplatelet treatment & $37(18.1)$ & $24(17.4)$ & 0.86 \\
\hline $\begin{array}{c}\text { Estimated } \\
\text { glomerular } \\
\text { filtration rate, } \\
\mathrm{ml} / \mathrm{min} ;\end{array}$ & $746 \pm 20.9$ & $83.4 \pm 20.2$ & 0.615 \\
\hline $\mathrm{Hb}, \mathrm{g} / \mathrm{dL}$ & $14.1 \pm 1.7$ & $14.5 \pm 1.4$ & 0.033 \\
\hline
\end{tabular}

Table 2 Events during follow-up

\begin{tabular}{|c|c|c|c|}
\hline \multirow{2}{*}{ Events } & VKAs & DOACs & \multirow{2}{*}{$\mathbf{p}$} \\
\cline { 2 - 3 } & $\mathbf{( n = 2 0 4 )}$ & $\mathbf{( n = 1 3 8 )}$ & \\
\hline Thromboembolism & $3(1.5 \%)$ & $0(0 \%)$ & 0.828 \\
\hline Bleedings & $27(13.2 \%)$ & $7(5.1 \%)$ & 0.017 \\
\hline Acute coronary syndrome & $2(1.0 \%)$ & $1(0.7 \%)$ & 1 \\
\hline Death & $4(2.0 \%)$ & $2(1.4 \%)$ & 0.763 \\
\hline At least 1 event & $36(17.2 \%)$ & $10(7.2 \%)$ & 0.012 \\
\hline
\end{tabular}

software SPSS version 15.0 for Windows (SPSS, Inc., Chicago, Illinois).

\section{Results}

During the inclusion period (june 2012 to june 2014), 342 patients with NVAF underwent ECV in the Arrhythmia Unit of Alicante General University Hospital. 138 patients (40.4\%) received DOACs and the rest, VKAs. The patient flow chart can be seen in Figure 1.

Baseline characteristics of the two groups of patients differed in several of the analyzed variables (Table 1). Patients who received VKA were older and had more comorbidities. The thromboembolic risk score CHA2DS2-VASc was lower in the DOACs group, with a median of 2 (IQR 1-3) compared to 3 (IQR 1-4) in the VKAs ( $\mathrm{p}<0.001$ ), as well as the HASBLED score, with a median of 1 (IQR 1-3) and 2 (IQR 1-3) respectively ( $\mathrm{p}<0.001$ ).

There were no thromboembolic events related to ECV (in the first 30 days after the procedure) in any of the groups.

At 1 year follow-up, all the events were more frequent in the VKA group (Table 2), but only the presentation of any type of bleeding showed significant differences among groups 
$(\mathrm{p}=0.017)$. The most frequent origin of bleeding was gastrointestinal (29.6\%). Four patients with VKA suffered major bleeding, compared to none in the DOACs group (14.8 vs $0 \%, \mathrm{p}=0.559$ ), all cases were extracranial hemorrhages.

The presence of at least one event was present in 45 patients (13.2\%), being more frequent in the VKAs group (17.2\%) compared to $7.2 \%$ in the DOACs group $(\mathrm{p}=0.012)$, with an absolute risk increase of $10.0 \%$ (95\% CI $=2.7$ to $16.5 \%)$. Survival graph until the first event can be seen in figure 2 .

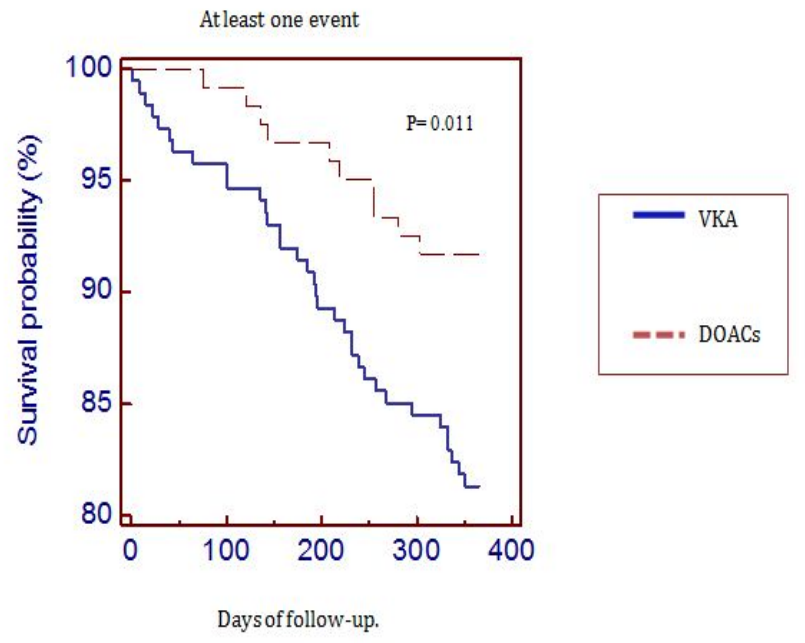

Figure 2: Survival graph until the first event (Kaplan meier curve)

Using multivariate analysis, the independent predictors of bleeding were hemoglobin (HR 0.77 [95\% CI 0.63-0.96]) and VKA use (HR 2.41 [95\% CI 1-5, 81]).

\section{Discussion}

DOACs are an increasingly used alternative in the management of patients with NVAF and are becoming a first-line tool in the context of ECV. In our series, DOACs were effective and safe for ECV in NVAF patients, without registering any early event. During mid-term follow-up, patients treated with DOACs had a significantly lower bleeding risk. Being a real-life population, our results are of great interest, because DOACs represent a simpler treatment to administer, with more stable anticoagulant activity, less interactions and less risk of intracranial bleeding than the classic treatment. In addition, DOACs have shown to decrease the required time interval to ECV [3], as the significant variability in anticoagulation levels in patients treated with VKAs makes it difficult to maintain the therapeutic range before the ECV, and this often forces to delay the procedure.

As we know, in many patients with persistent AF, ECV is a fast and efficient mechanism to restore sinus rhythm, but it requires oral anticoagulation for at least 3 weeks in patients with AF lasting more than 48 hours. In non-anticoagulated patients the periprocedure risk of ischemic event associated with ECV is between 5 and $7 \%$, but warfarin treatment reduces the incidence of thromboembolic events between 0.5 and 1.6\% [6]. International guide- lines recommend at least 3 consecutive weeks of effective anticoagulation prior to cardioversion, followed by at least another 4 weeks of anticoagulation thereafter $[5,7]$.

The X-VeRT study randomized individuals with NVAF to rivaroxaban or warfarin prior to ECV [3]. In this study, along with analysis of the RE-LY and ARISTOTLE studies [1, 2], and similarly to our study, the incidence of systemic stroke or embolism at 30 days of ECV was less than 1\%, with no difference from the type of anticoagulant therapy received.

At mid-term follow-up, the rate of thromboembolic events of our series was similar of pivotal studies, being in these a $1.3 \%$ in VKA group and 0\% in DOAC group; on the other hand, the rate of bleeding of any type was greater in VKA group (14.5\%) than in DOAC group (5.8\%), with only four major bleedings, all of them extracranial, in the group with VKA; in contrast, in pivotal trials with DOACs, these had a higher bleeding rate than warfarin at expense of digestive bleeding, but less incidence of severe and intracranial bleeding.

Our main limitation is inherent to the design of the study, since it is an observational study, the treatment groups were not randomized, but the allocation of treatment with VKAs or DOACs was performed by the cardiologist who attended the patient previously. Therefore, the study groups were not similar with respect to some of their baseline characteristics. The greater comorbidity of the patients under treatment with VKA could explain, at least in part, the increase of hemorrhagic and thromboembolic events in the follow-up in this subgroup. It should be noted that this is a study of real-life patients and we have found that, during the inclusion period, DOACs were used in people with less comorbidities, probably due to physicians initial distrust on these new treatments. However, a multivariate analysis was performed to control the heterogeneity of the groups under study. Despite these limitations, we believe that the conclusions of the study are of interest for daily clinical practice.

\section{Conclusion}

In a real population of individuals with NVAF undergoing ECV, DOACs were effective in prevention of short and mid-term thromboembolic events, showing also an adequate safety profile.

\section{References}

1. Nagarakanti R, Ezekowitz MD, Oldgren J, Yang S, Chernick M, Aikens TH, et al. Dabigatran versus warfarin in patientwith atrial fibrillation: ananalysis of patients undergoing cardioversion. Circulation. 2011;123(2):131-136. doi: 10.1161/CIRCULATIONAHA.110.977546.

2. Flaker G, Lopes RD, Al-Khatib SM, Hermosillo AG, Hohnloser $\mathrm{SH}$, Tinga B, et al. Efficacy and safety of apixaban in patients after cardioversion for atrial fibrillation: in sights from the ARISTOTLE trial (apixaban for reduction in stroke and other thromboembolic events in atrial fibrillation). J Am Coll Cardiol. 2014;63(11):1082-1087. doi: 10.1016/j.jacc.2013.09.062

3. Cappato R, Ezekowitz MD, Klein AL, Camm AJ, Ma CS, Le Heuzey JY, et al. Rivaroxaban vs. vitamin $\mathrm{K}$ antagonists for cardioversion in atrial fibrillation. Eur Heart J. 2014;35(47):3346-3355. doi: 10.1093/eurheartj/ehu367 
4. Santas E, Mendez J, Martinez-Brotons A, Nuñez J, Chorro FJ, Ruiz-Granell R. Experience in daily clinical practice of ambulatory cardioversión of atrial fibrillation treated with new oral anticoagulants. Rev Esp Cardiol. 2014;67(11):960-961. doi: 10.1016/j.rec.2014.06.019

5. Camm AJ, Lip GYH, Caterina RD, Savelieva I, Atar D, Hohnloser SH, et al. 2012 focused update of the ESC Guidelines for the management of atrial fibrillation An update of the 2010 ESC Guidelines for the management of atrial fibrillation Developed with the special contribution of the European Heart Rhythm Association. Europace. 2012; 14(10):1385-1413
6. Bjerkelund CJ, Orning OM. The efficacy of anticoagulant therapy in preventing embolism related to D. C. electrical conversion of atrial fibrillation. Am J Cardiol. 1969;23(2):208216.

7. Arnold AZ, Mick MJ, Mazurek RP, Loop FD, Trohman RG. Role of prophylactic anticoagulation for direct current cardioversion in patients with atrial fibrillation or atrial flutter. $\mathrm{J}$ Am Coll Cardiol. 1992;19(4):851-855. 